\title{
Numerical Study of Predator-Prey Model with Beddington-DeAngelis \\ Functional Response and Prey Harvesting
}

Fira Fitriah ${ }^{1}$, Agus Suryanto ${ }^{1^{*}}$, Noor Hidayat ${ }^{2}$

${ }^{1}$ Department of Mathematics, Faculty of Mathematics and Sciences, Universitas Brawijaya, 65145, Malang, Indonesia

\section{ABSTRACT}

A modified Leslie-Gower predator-prey model with Beddington-DeAngelis functional response and Michaelis-Menten type prey harvesting is studied. The equilibrium points of the system are investigated. To see the stability of each equilibrium point, we perform some numerical simulations. Our numerical simulations show that the extinction of prey or survival of both prey and predator are conditionally stable.

Keywords: harvesting, Leslie-Gower, Michaelis-Menten, numerical simulations, predator-prey model

\section{INTRODUCTION}

Every species interact with other species in their habitat. Interactions between species lead to a rise or reduction population densities. Predation is one form of interaction between the two species (predator and prey). In the case of predation, a predator population density is increasing. In contrast, the density of the prey population is decreasing. Species compete, evolve, and spread for the aim of seeking the source of food to sustain their existence [1].

Environmental resources constrain the growth of each species. If the population density increases, the growth rate of population is reduced and stopped at a certain population density that is known as the carrying capacity. In other words, carrying capacity is the maximum population density that can be supported by the environment [2].

In population dynamics, a functional response represents the predation rate of prey by predators per capita. Holling [3] has introduced a functional response that depends only on the prey species, namely Holling type I, II, and III. Beddington [4] and DeAngelis [5] have modified the Holling functional response. Beddington and DeAngelis suggested that predation rate is more realistic if it also depends on the predator species and environmental provides protection to the prey. Predator population densities are important to consider because the predators are competing

\footnotetext{
*Corresponding author:

Agus Suryanto

Department of Mathematics, Faculty of Mathematics and Science, Universitas Brawijaya, 65145, Malang, Indonesia

E-mail: suryanto@ub.ac.id
}

and sharing food.

Leslie [6] has introduced a predator-prey model with carrying capacity of the predator population is proportional to the number of prey population. The model is known as the Leslie-Gower predator-prey model. Aziz-Alaoui and Okiye [7] has modified the carrying capacity of the predator population in LeslieGower model. Aziz-Alaoui and Okiye involved the environment protection. They used Holling-type II functional responses. The existence of the economic value of the prey population encourages modification by adding prey harvesting. Gupta and Chandra [8] has modified Aziz-Alaoui and Okiye model by incorporating Michaelis-Menten type prey harvesting. Yu [9] has modified Aziz-Alaoui and Okiye model by the functional response into Beddington-DeAngelis-type.

In this article, we extend the predator-prey model proposed by Gupta and Chandra [8] and Yu [9] by incorporating a Bedington-DeAngelis functional response and the Michaelis-Menten type prey harvesting. The focus of this study is to give an overview of population densities in the long term based on numerical simulations. Fourth order Runge-Kutta method is used to solve the predator-prey system.

\section{MATERIALS AND METHODS Mathematical model}

A mathematical model that represents the interaction between the prey species and the predator species will be constructed based on several assumptions. First, we assume that harvesting is performed only on prey species. Second, the coefficients of environmental protection for the prey and predator population are differ- 
ent.

Suppose $x$ is the population density of prey and $y$ is the population density of predator. It is assumed that prey grows with intrinsic growth rate $r_{1}$ :

$$
\frac{d x}{d t}=r_{1} x
$$

The existence of competition among prey species to survive in their habitat with competition rate $p$ causing the prey population growth rate was reduced as follow

$$
\frac{d x}{d t}=-p x^{2}
$$

Based on $\mathrm{Yu}$ [9], the rate of predation is given in the form of the Beddington-DeAngelis functional response:

$$
p(x, y)=\frac{\alpha}{a+b x+c y},
$$

where $\alpha$ and $a$ are the maximum value of the per capita reduction rate of $x$ due to $y$ and the coefficient of environmental protection for the prey, respectively. $\mathrm{b}$ and $\mathrm{c}$ are suitable constants. Therefore, the prey population growth rate is reduced due to predation as follow

$$
\frac{d x}{d t}=-\frac{\alpha x y}{a+b x+c y}
$$

Harvesting prey by humans given in a nonlinear form, known as the Michaelis-Menten type as in Gupta and Chandra [8]. The prey population growth rate is reduced due to harvesting as follow where $q$ is catchability coefficient, $E$ is the effort

$$
\frac{d x}{d t}=-\frac{q E x}{m_{1} E+m_{2} x},
$$

applied to harvest the prey species, and $m_{1}$ and $m_{2}$ are suitable constants. $m_{1} E$ shows limited human effort to harvest and $m_{2} \mathrm{X}$ indicates the limited number of prey that harvested. Based on the equation (1), (2), (3), and (4), the prey population growth rate is formulated as follow with all parameters are positive constant.

$$
\begin{aligned}
\frac{d x}{d t}= & r_{1} x-p x^{2}-\frac{\alpha x y}{a+b x+c y} \\
& -\frac{q E x}{m_{1} E+m_{2} x},
\end{aligned}
$$

It is assumed that predator grows with intrinsic growth rate $r_{2}$ :

$$
\frac{d y}{d t}=r_{2} y
$$

As Aziz-Alaoui and Okiye [7], the carrying capacity of the predator population is limited by prey population density and the extent to which the environmental provides protection to predator ( $k$ ). If $\beta$ is the competition rate among predator species to survive in their habitat, the predator population growth rate is reduced as follow

$$
\frac{d y}{d t}=-\frac{\beta y^{2}}{x+k}
$$

Based on the equation (5) and (6), the predator population growth rate is formulated as follow

$$
\frac{d y}{d t}=y\left(r_{2}-\frac{\beta y}{x+k}\right)
$$

Thus, predator-prey models involving BeddingtonDeAngelis functional response and Michaelis-Menten type prey harvesting is

$$
\begin{aligned}
\frac{d x}{d t}= & r_{1} x-p x^{2}-\frac{\alpha x y}{a+b x+c y} \\
& -\frac{q E x}{m_{1} E+m_{2} x}, \\
\frac{d y}{d t}= & y\left(r_{2}-\frac{\beta y}{x+k}\right) .
\end{aligned}
$$

\section{Determination of the equilibrium point}

The equilibrium point of the system (7) is the solution of that system when both population growth rates are zero. Equilibrium point illustrates a constant solution of the system.

\section{Observing the stability of the equilibrium point}

Stability of the equilibrium point is observed by the Jacobian matrix of the system. The stability property shows whether the equilibrium point is stable or not. If the equilibrium point is stable, then any solution of the system with different initial values will be convergent to it, and vice versa.

\section{Numerical experiments}

System (7) is a nonlinear system, so the analytical solution of system (7) is not easy to be determined. Therefore, numerical simulations will be performed to investigate the system behavior. Numerical solutions of the system can give an overview of population densities 
in the long term. To determine the numerical solutions of the predator-prey model, we use the fourth order Runge-Kutta method.

\section{RESULTS AND DISCUSSION \\ Equilibrium Point} (7), let

In order to find the equilibrium point of the system

$$
\begin{aligned}
& 0=\left(r_{1}-p x-\frac{\alpha y}{a+b x+c y}-\frac{q E}{m_{1} E+m_{2} x}\right) x, \\
& 0=\left(r_{2}-\frac{\beta y}{x+k}\right) y,
\end{aligned}
$$

where $x$ and $y$ have same meaning as $x(t)$ and $y(t)$, respectively. Obviously, system (7) has five equilibrium points, as follow.

i. The extinction of both prey and predator point $E_{0}=(0,0)$

ii. The predator extinction point $E_{1}=\left(x_{A}^{*}, 0\right)$ and $E_{2}=\left(x_{B}^{*}, 0\right)$ where

$$
\begin{gathered}
\Delta_{1}=\left(m_{2} r_{1}-m_{1} p E\right)^{2}+4 m_{2} p\left(m_{1} r_{1} E-q E\right), \\
x_{A}^{*}=\frac{1}{2}\left(\frac{m_{2} r_{1}-m_{1} p E+\sqrt{\Delta_{1}}}{m_{2} p}\right), \\
x_{B}^{*}=\frac{1}{2}\left(\frac{m_{2} r_{1}-m_{1} p E-\sqrt{\Delta_{1}}}{m_{2} p}\right) .
\end{gathered}
$$

iii. The prey extinction point $E_{3}=\left(0, r_{2} \mathrm{k} / \beta\right)$

iv. The positive equilibrium point $E_{4}\left(x^{*}, r_{2}\left(x^{*}+\right.\right.$ $k) / \beta$ ). $x^{*}$ is a positive real root of

$$
\left(x^{*}\right)^{3}+\frac{B}{A}\left(x^{*}\right)^{2}+\frac{C}{A} x^{*}+\frac{D}{A}=0,
$$

where

$$
\begin{aligned}
u & =a \beta+c r_{2} k, \\
v & =b \beta+c r_{2}, \\
A & =-m_{2} p v \\
B & =m_{2}\left(r_{1} v-p u\right)-m_{1} E p v-m_{2} \alpha r_{2}, \\
C & =r_{1} m_{2} u+m_{1} E\left(r_{1} v-p u\right)-q E v \\
\quad & \quad-\alpha r_{2}\left(k m_{2}+m_{1} E\right), \\
D= & r_{1} u m_{1} E-\alpha r_{2} k m_{1} E-q E u .
\end{aligned}
$$

In contrast to all of the equilibrium point, $E_{4}\left(x^{*}, r_{2}\right.$ $\left.\left(x^{*}+k\right) / \beta\right)$ shows that both the prey and the predator can survive in the long term.

\section{Stability of Equilibrium Point}

The Jacobian matrix of system (7) at equilibrium point $(\mathrm{x}, \mathrm{y})$ is

$$
J=\left[\begin{array}{ll}
a_{11} & a_{12} \\
a_{21} & a_{22}
\end{array}\right]
$$

with

$$
\begin{aligned}
& a_{11}=r_{1}-2 p \hat{x}-\frac{\alpha \hat{y}(a+c \hat{y})}{(a+b \hat{x}+c \hat{y})^{2}}-\frac{q m_{1} E^{2}}{\left(m_{1} E+m_{2} \hat{x}\right)^{2}}, \\
& a_{12}=-\frac{\alpha \hat{x}(a+b \hat{x})}{(a+b \hat{x}+c \hat{y})^{2}}, \\
& a_{21}=\frac{\beta \hat{y}^{2}}{(\hat{x}+k)^{2}} \\
& a_{22}=r_{2}-\frac{2 \beta \hat{y}}{\hat{x}+k} .
\end{aligned}
$$

An equilibrium point ( $x, y)$ of system (7) is asymtotically stable if the real parts of all eigenvalues of $J(\mathrm{x}, \mathrm{y})$ are negative. The Jacobian matrices for $E_{0}, E_{1}$, $E_{2}$ and $E_{3}$ are

$$
\begin{aligned}
& J_{0}=\left(\begin{array}{cc}
r_{1}-\frac{q}{m_{1}} & 0 \\
0 & r_{2}
\end{array}\right), \\
& J_{1}=\left(\begin{array}{cc}
r_{1}-2 p x_{A}^{*}-\frac{q m_{1} E^{2}}{\left(m_{1} E+m_{2} x_{A}^{*}\right)^{2}} & -\frac{\alpha x_{A}^{*}}{a+b x_{A}^{*}} \\
0 & r_{2}
\end{array}\right), \\
& J_{2}=\left(\begin{array}{cc}
r_{1}-2 p x_{B}^{*}-\frac{q m_{1} E^{2}}{\left(m_{1} E+m_{2} x_{B}^{*}\right)^{2}} & -\frac{\alpha x_{B}^{*}}{a+b x_{B}^{*}} \\
0 & r_{2}
\end{array}\right), \\
& J_{3}=\left(\begin{array}{cc}
r_{1}-\frac{\alpha r_{2} k}{a \beta+c r_{2} k}-\frac{q}{m_{1}} & 0 \\
\frac{r_{2}^{2}}{\beta} & -r_{2}
\end{array}\right),
\end{aligned}
$$

respectively. $r_{2}$ is a positive eigenvalue of $J_{0^{\prime}} J_{1}$ and $J_{2}$, so $E_{0}, E_{1}$ and $E_{2}$ are unstable equilibrium point. Therefore, the extinction of both prey and predator or predator extinction does not occur in the long term. We next investigate the stability of $E_{3}$ and $E_{4}$ by numerical simulations.

\section{Numerical Simulations}

Let $r_{1}=0.3, \mathrm{a}=10, b=1, c=0.5, q=0.3, E=10, p=$ $0.05, m_{1}=0.1, \quad \alpha=0.02, m_{2}=0.6, r_{2}=0.2, \quad \beta=0.5$ and

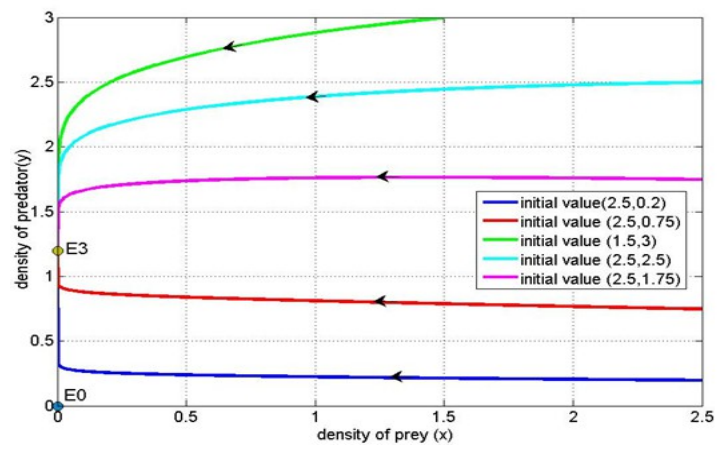

Figure 1. Numerical solution of system (7) with an asymptotically stable equilibrium point $E_{3}$ 
$k=3$. Based on those parameter values, the equilibrium point $E_{0}$ and $E_{3}$ are exist. The values of each point of equilibrium are $E_{0}=(0,0)$ and $E_{3}=(0,1.2)$. The eigenvalues of $J\left(E_{0}\right)$ are -2.7 and 0.2 . Whereas, $J\left(E_{3}\right)$ the eigenvalues of are -0.2 and -2.70226 . As a consequnce of stability requirements, is unstable $E_{0}$ and $E_{3}$ is an asymptotically stable. These results are shown in Figure 1.

The initial values in Figure 1 show the initial den sity of both prey and predator. For various initial values, all numerical solutions of system (7) converge to $E_{3}$. In this numerical simulation, we choose small values of $m_{1}$ and $m_{2}$ which indicate the large prey harvesting. Clearly that relatively large harvesting leads to prey extinction. On the other hand, predators still survive in the habitat because there is a high environmental protection $(k)$. The environmental protection includes the temperature, humidity, or other abiotic factors in the habitat. Without prey in the habitat, predators grow until it reaches carrying capacity $\left(r_{2} k / \beta\right)$. Therefore, the prey extinction are predicted to occur in the long term when there is a large prey harvesting.

For the second simulation, we use the same parameter values as before except for parameter $m_{1}$ and $m_{2}$. In this simulation, the values of $m_{1}$ and $m_{2}$ are respectively 1.07 and 2 . There are four equilibrium points i.e. $E_{0}=(0,0), E_{1}=(1.8101,0), E_{3}=(0,1.2)$ and $E_{4}=$ $(1.66299,1.86519)$. The eigenvalues of $J\left(E_{0}\right)$ are 0.0196 and 0.2 , the eigenvalues of $J\left(E_{1}\right)$ are -0.03754 and 0.2 , and the eigenvalues of $J\left(E_{3}\right)$ are -0.2 and -0.01736 . On the other hand, the eigenvalues of $J\left(E_{4}\right)$ are -0.03321 and -0.198827 .

Therefore, $E_{0}, E_{1}$ and $E_{3}$ are unstable and $E_{4}$ is asymptotically stable. In Figure 2, we plot some numerical solutions using several initial values. All numerical solutions of system (7) converge to $E_{4}$. Note that in this simulation, we use greater values of $m_{1}$ and $m_{2}$, meaning that the harvesting is decreased. Consequently, prey can survive although the intrinsic growth rate of prey is low. The presence of food (prey) causes predator to survive. Therefore, predator and prey can coexist in the long term if prey harvesting is reduced.

\section{CONCLUSIONS}

In this paper, we have studied a modified LeslieGower predator-prey model with Beddington-DeAngelis functional response and Michaelis-Menten type prey harvesting numerically. It is found that the model has five equilibrium points $\left(E_{0}\right)$, namely the extinction of both prey and predator point the predator extinction point $\left(E_{1}\right.$ and $\left.E_{2}\right)$, the prey extinction point $\left(E_{3}\right)$, and

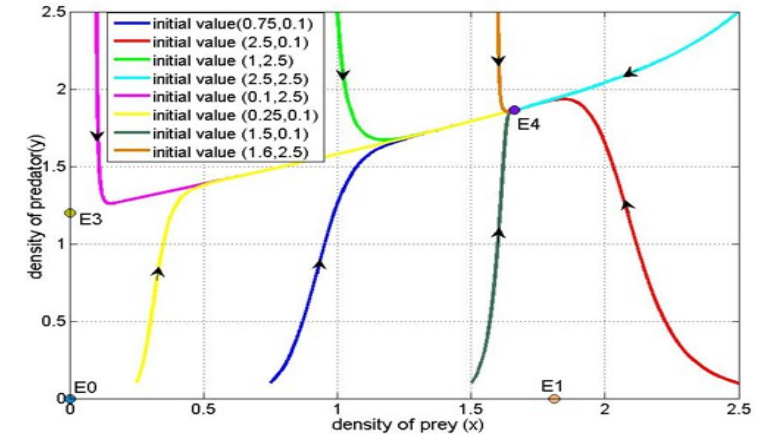

Figure 2. Numerical solution of system (7) with an asymptotically stable equilibrium point $E_{4}$

the survival of both prey and predator point $\left(E_{4}\right)$. Based on numerical simulations, there are two possible stable equilibrium points that are $E_{3}$ and $\mathrm{E}_{4} . E_{3}$ and $E_{4}$ respectively indicated the prey extinction or the survival both prey and predator in the long term. In the future, we will explore the dynamics of the model analytically.

\section{REFERENCES}

1. Campbell NA, Reece JB, Mitchell LG (2004) Biologi Vol. 3. 5th Ed. Erlangga. Jakarta.

2. Molles MC (2002) Ecology Concept and Applications. 2nd Edition. Mc Graw Hill. Mexico City.

3. Holling CS (1965) The Functional Response of Predator to Prey Density and its Role in Mimicry and Population Regulation. Memoirs of the Entomological Society of Canada. 97: 5-60.

4. Beddington JR (1975) Mutual Interference between Parasites or Predators and its Effect on Searching Efficiency. Journal of Animal Ecology. 44: 331-340.

5. DeAngelis DL, Goldstein RA, O’Neill RV (1975) A Model of Trophic Interaction. Ecology. 56: 881892.

6. Leslie PH (1948) Some Further Notes on the Use of Matrices in Population Mathematics. Biometrika. 35: 213-245.

7. Aziz-Alaoui MA, Okiye MD (2003) Boundedness and Global Stability for a Predator-Prey Model with Modified Leslie-Gower and Holling-type II schemes. Applied Mathemathics Letters. 16: 10691075.

8. Gupta RP, Chandra P (2013) Bifurcation Analysis of a Modified Leslie-Gower Predator-Prey Model with Michaelis-Menten type Prey Harvesting. Journal of Mathematical Analysis and Application. 398: 278-295. 
9. Yu S (2014) Global Stability of a Modified LeslieGower Model with Beddington-DeAngelis Functional Response. Advances in Difference Equa tions. 84: 1-14. 